\title{
Dissociating Cognitive and Motoric Precursors of Human Self-Initiated Action
}

\author{
Nima Khalighinejad*, Elisa Brann**, Alexander Dorgham, and Patrick Haggard
}

\begin{abstract}
Across-trial variability of EEG decreases more markedly before self-initiated than before externally triggered actions, providing a novel neural precursor for volitional action. However, it remains unclear whether this neural convergence is an early, deliberative stage or a late, execution-related stage in the chain of cognitive processes that transform intentions to actions. We report two experiments addressing these questions. Participants viewed randomly moving dots on a screen. At a random time, all dots started moving coherently to the left or right side of the screen. Participants were rewarded for correctly responding to the direction of coherent dot movement. However, the waiting time before coherent dot motion onset could be extremely long. Participants had the option to skip waiting by pressing a "skip" key. These self-initiated "skips"
\end{abstract}

\section{INTRODUCTION}

Our everyday actions span a spectrum of autonomy, ranging from simple reflexes, immediate motor responses to external stimuli, to complex volitional actions, which are not directly determined by any identifiable external stimulus (Haggard, 2008). Functional and neuroanatomical studies have helped to refine this spectrum, identifying specific neural differences between selfinitiated and externally triggered actions (Passingham, Bengtsson, \& Lau, 2010; Cunnington, Windischberger, Deecke, \& Moser, 2003; Jenkins, Jahanshahi, Jueptner, Passingham, \& Brooks, 2000; Deiber, Honda, Ibañez, Sadato, \& Hallett, 1999; Passingham, 1987). An action that involves directly responding to an external cue, such as a choice RT, or responding to a verbal command, can be considered exogenous or "externally triggered." In contrast, an endogenous, self-initiated action requires no external cue: The agent decides for themselves whether and when to act. Depending on the situation in which they are made, self-initiated actions often have additional

\footnotetext{
University College London

*N. K. is now at the Wellcome Trust Centre for Integrative Neuroimaging, Department of Experimental Psychology, University of Oxford, Oxford, United Kingdom.

**E. B. is now at the Institute of Psychiatry, King's College London, London, United Kingdom.
}

were compared with blocks where participants were instructed to skip. EEG variability decreased more markedly before selfinitiated compared with externally triggered "skip" actions, replicating previous findings. Importantly, this EEG convergence was stronger at frontomidline electrodes than at either the electrode contralateral or ipsilateral to the hand assigned to the "skip" action in each block (Experiment 1). Furthermore, convergence was stronger when availability of skip responses was "rationed," encouraging deliberate planning before skipping (Experiment 2). This suggests that the initiation of voluntary actions involves a bilaterally distributed, effectorindependent process related to deliberation. A consistent process of volition is detectable during early, deliberative planning and not only during late, execution-related time windows. distinctive properties linking them to voluntariness and individual autonomy. For example, self-initiated actions are often susceptible to reason (Anscombe, 2000), free from immediacy (Gold \& Shadlen, 2007), and often involve choosing between alternatives (Pereboom, 2011).

The readiness potential (RP), also known as the bereitschaftspotential, is a gradual build-up of electrical potential in the premotor areas that occurs before selfinitiated movements (Kornhuber \& Deecke, 1965), often beginning $1 \mathrm{sec}$ or more before movement onset (Haggard, 2008). Classically, RP is taken to be the electrophysiological sign of planning, preparation, and initiation of voluntary actions (Kornhuber \& Deecke, 1990) and was suggested as the neural precursor to conscious intention to act (Libet, Gleason, Wright, \& Pearl, 1983).

The view that RP reflects a fixed precursor process that leads to self-initiated, voluntary action has been recently challenged (Schurger, Mylopoulos, \& Rosenthal, 2016; Schurger, Sitt, \& Dehaene, 2012). These new models suggest that rising ramp pattern of the mean RP does not reflect a specific goal-directed process but rather reflect subthreshold fluctuations in premotor activity that influence the precise time of the action (Murakami, Vicente, Costa, \& Mainen, 2014; Schurger et al., 2012). The negative shape of the RP is not a readout of a specific preparatory process but results from cross-trial averaging of these stochastic fluctuations time-locked to action initiation, a common practice in computing mean RP. 
Given the current debate on neural precursors of human voluntary action, we recently calculated the time course of variability across trials in the RP signal. Classical models, which view the RP as marking a distinctive cognitive process preceding self-initiated actions, seem committed to low EEG variability during the RP period. In contrast, stochastic models would imply higher variability during the same period, with the proviso that variability must inevitably decrease before action if the trigger for action indeed involves a neural signal approaching a threshold value. In any such model, variability will always decrease before action because the random walk can only approach the trigger threshold from below. This decrease is either an artifact or a logical consequence of action-locked epoching. Crucially, this decrease should be the same in all conditions where the random walk is responsible for the trigger, assuming the stochastic process producing the random walk does not itself change. Thus, the timing and magnitude of EEG variability before action can be informative about the neural processes that generate action. We indeed observed that across-trial variability of EEG decreased before action initiation. Furthermore, this decrease was more marked before endogenous, self-initiated actions compared with externally triggered actions (Khalighinejad, Schurger, Desantis, Zmigrod, \& Haggard, 2018). We showed that, in addition to stochastic fluctuation in neural activity, a process of noise control before self-initiated actions may consistently contribute to decision time to move in humans (Khalighinejad et al., 2018). Importantly, this process could be observed as across-trial convergence of neural activity recorded by scalp electrodes from premotor cortex. However, it is not clear whether this neural marker of self-initiated action represents an effectorindependent, general neurocognitive process of volition or an effector-specific motoric activity.

RP begins symmetrically. However, before movement, the RP lateralizes, with stronger amplitudes observed over the hemisphere contralateral to the effector performing the movement (Eimer, 1998). This lateralized RP (LRP) reflects preparation to execute an action in an effector-specific manner (e.g., which hand will be used to press the button; Kutas \& Donchin, 1980). In serial models of action control, LRP onset represents a useful dividing line between earlier ends and later means, separating the cognitive mechanisms of preparing actions, from the motor mechanisms related to the specific movement that implements the action goal. Here, we ask whether the EEG convergence that precedes self-initiated action reflects an early cognitive, effectorindependent process or rather reflects a motoric, effector-dependent process.

To answer this question, we performed two experiments using a modified version of a recently developed paradigm (Khalighinejad et al., 2018). In the original task, participants responded to the direction of unpredictably occurring dot motion stimuli by pressing either the left or right arrow keys. Importantly, they could also choose to skip waiting for the stimuli to appear by pressing both keys simultaneously whenever they wished. The skip response thus reflected a purely endogenous decision to act, without any direct external stimulus, and provided an operational definition of a self-initiated action. The self-initiated skip responses were compared with an externally triggered skip block in which participants made the same skip action in response to an unpredictable visual cue, as opposed to whenever they wished. In the first experiment, we required participants to use either the left or right hand, in separate blocks, to perform the skip response. This modification enabled us to record EEG convergence contralateral to the acting hand. If our putative signal of self-initiated action is effector-specific, we would expect to see a stronger convergence at EEG electrodes contralateral to the hand assigned to perform the self-initiated skip response. Alternatively, if the signal is effector-independent, we would expect to see a stronger EEG convergence at midline electrodes, regardless of the hand used for skip response. This manipulation effectively probes the role of motoric execution-related processes in the initiation and elaboration of voluntary action.

In the second experiment, we "rationed" volition by restricting the number of self-initiated skip actions that could be made. We reasoned that this "rationing" encourages careful and deliberate planning of self-initiated actions compared with a condition where "skip" actions are unlimited. The logic was as before. If we find stronger convergence in the more deliberate, rationed condition, we would conclude that such convergence reflects cognitive processes that deliberate the likely value of action.

\section{METHODS}

\section{Participants}

For sample size calculation, we performed power analysis (Faul, Erdfelder, Lang, \& Buchner, 2007) with an estimated effect size of 0.7 based on our previous results, alpha of .05 and power of 0.85 . This yielded total sample size of 21 for each experiment. Forty-five right-handed participants (23 for Experiment 1 and 22 for Experiment 2) aged $18-42$ years old ( 17 men, mean age $=24.6$ years $)$ were recruited via the University College London Institute of Cognitive Neuroscience participant data pool. Six participants were excluded (three from each study) before data analysis because of poor EEG quality. The final sample size was 20 participants for Experiment 1 and 19 for Experiment 2. All participants fulfilled the recruitment requirements, including no history of psychiatric and/or neurological disorders, no brain stimulation $48 \mathrm{hr}$ before the study, having normal or corrected-to-normal vision, and no color blindness. Participants received payment for their time based on an institution-approved 
hourly rate. The experimental design and procedure were approved by the University College London research ethics committee and followed the principles of the Declaration of Helsinki.

\section{Behavioral Task and Procedure}

Following consent, participants sat in front of a computer screen $(60 \mathrm{~Hz}$ refresh rate), in an electrically shielded chamber, and were fitted with the EEG cap. Participants were given verbal instructions and performed two practice blocks of trials to familiarize themselves with the task.

The behavioral task was adapted from our previous study (Khalighinejad et al., 2018). In brief, participants were required to focus on a fixation point in the center of the screen. At the beginning of each trial, the fixation point was black but gradually and randomly changed color. Meanwhile, participants observed randomly moving dots displayed within a circular aperture of $7^{\circ}$ in diameter (density of 14.28 dots/degree, initially moving with $0 \%$ coherence with a speed of $2 \%$ sec; Desantis, Waszak, \& Gorea, 2016; Desantis, Waszak, Moutsopoulou, \& Haggard, 2016). Participants were instructed to wait until all dots moved in the same direction (step change to
100\% coherence). The dots moved either to the left or right of the midline ranging in their degree of discrimination difficulty, with dot movement oriented upward being more difficult to discriminate compared with sideways movements. The participant's task was to determine the direction of dot motion, responding using the appropriate left ("D") or right ("L") keys on the keyboard with their respective left or right index finger. If participants responded correctly, they were rewarded (2 pence), whereas incorrect responses resulted in a penalty ( -1 pence). Responses that were early (before coherent dot motion) or delayed (after $2 \mathrm{sec}$ of coherent dot motion) also received a penalty ( -1 pence) and were followed by an error message (Figure 1).

Each experimental session was limited to $40 \mathrm{~min}$. Participants were informed that the elapsing time between trial onset and coherent dot motion onset was random (drawn from an exponential distribution with $\min =$ $2 \mathrm{sec}, \max =60 \mathrm{sec}$, mean $=12 \mathrm{sec}$ ), and consequently, the waiting time could be very long. To avoid long waiting times, participants had the option to skip from one trial to the next by pressing the "space" bar with their left or right thumb or both (depending on the experiment; see later) and claiming a smaller reward (1 pence). They were reminded that they should consider the
Figure 1. Timeline of an experimental trial. Participants responded to the direction of dot motion with left and right keypresses. Dot motion could begin unpredictably, after a delay drawn from an exponential distribution. (A) In the "self-initiated" blocks, participants waited for an unpredictably occurring dot motion stimulus and were rewarded for correct left-right responses to motion direction. They could decide to skip long waits for the motion stimulus by pressing the space bar with the left or right thumb (Experiment 1) or making a bilateral keypress (Experiment 2). They thus decided between waiting, which lost time but brought a large reward, and "skipping," which saved time but brought smaller rewards. The color of the fixation cross changed continuously during the trial but was irrelevant to the decision task. (B) In the "externally triggered" blocks, participants were instructed to press the space bar with left or right thumb (Experiment 1) or both (Experiment 2) when the fixation cross became red, and not otherwise.

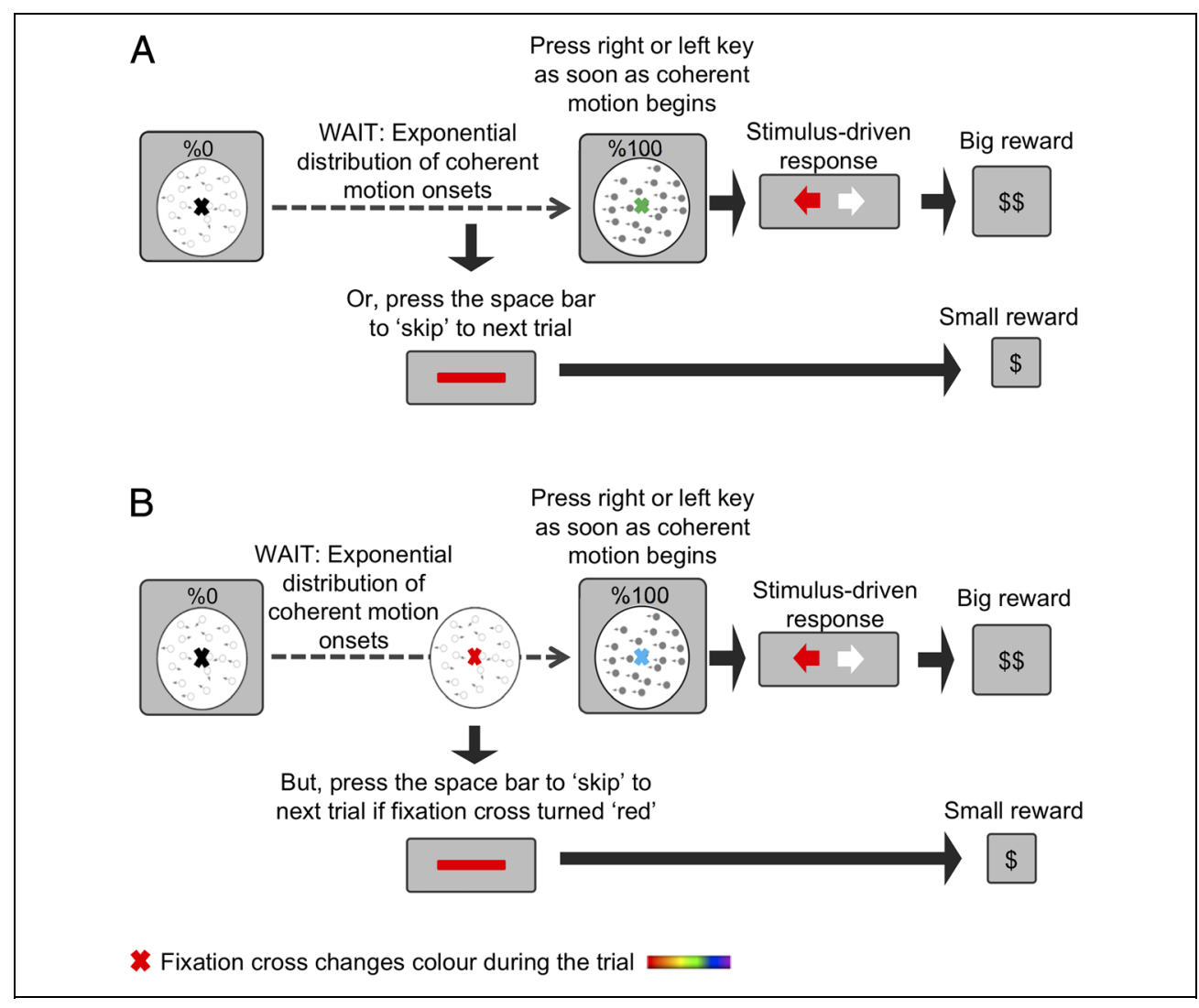


time-money trade-off in each trial. They could win a big reward by waiting, if responding correctly, but losing time or could save time by skipping and collecting more guaranteed smaller rewards.

The task involved two alternating blocks that varied in skipping behavior. In the "self-initiated" blocks (Figure 1A), participants could skip at any moment of their choosing following the onset of the trial. This skip response thus reflects a purely endogenous decision to act in the absence of any external instruction to act and based on the trade-off between later, larger and smaller, earlier rewards. This provides an operational definition of selfinitiated action within our experimental design. In the "externally triggered" blocks (Figure 1B), participants had to skip in response to an external cue. The external cue was an unpredictable change in the color of the fixation point to red. The color cycle of the fixation cross had a random sequence, and the timing of appearance of the red color was yoked to the time of participant's own previous skip responses in the immediately preceding self-initiated block. If participants skipped correctly in response to the color cue, they were rewarded (1 pence); however, they received a penalty ( -1 pence) if they skipped too early (before the fixation point turned red) or too late (after $2 \mathrm{sec}$ of the fixation point being red). Thus, in "externally triggered" condition blocks, participants could not choose for themselves when to skip. To control for any confounding effect of attending to fixation point, participants were also required to monitor its color in the self-initiated blocks and to roughly estimate the number of times the fixation point turned yellow, according to the following categories: never, less than 50\%, 50\%, more than 50\%. The red color was left out of the color cycle in the self-initiated blocks.

At the end of each block, participants received feedback on their reward values, total elapsed time, and number of skips, enabling them to adjust their behavior over time and maximize earnings. Each block consisted of 10 trials, and the order of the blocks was counterbalanced across participants.

In Experiment 1, participants used either the left or right thumb to perform the skip action. Once $40 \mathrm{~min}$ passed using one thumb, the task finished, and participants repeated the same task, after 5 min break, but using the other thumb to skip. Skipping hand order (right-left or left-right) was counterbalanced across participants. To ensure sufficient data collection and to avoid long testing sessions, the experiment was split across two sessions, which took place on different days. Data from similar conditions were pooled across the two sessions. Thus, the experiment had two factors: the hand used for skipping (left vs. right), and the type of skip action (self-initiated vs. externally triggered). To perform the skip action in Experiment 2, participants used both thumbs simultaneously. Experiment 2 consisted of two sessions: In the first session (unlimited session), similar to Experiment 1, there was no limitation in the number of skip actions participants could perform in the selfinitiated blocks. In the second session (limited session), however, they were informed that they could only make half the number of skips made in the first session. For example, if a participant skipped waiting 100 times in the first session, they were allowed to skip max 50 times in the second session. Total number of allowed skips was displayed on the screen at the beginning of the session, and they were informed of the number of remaining skips at the end of each block. If a participant used all their allowed skip actions before the end of the experiment, the experiment continued but they were not allowed to skip anymore and had to wait for coherent dot motion before responding. They were not rewarded for saving their skip actions. The behavioral task was designed in Psychophysics Toolbox Version 3 (Brainard, 1997).

\section{EEG Recording}

The experiment was conducted inside an electrically shielded chamber. EEG signals were recorded and amplified using an ActiveTwo Biosemi system. Participants wore a 64-channel EEG cap. To reduce preparation time, they were only fitted with a subset of 20 electrodes covering the central and visual areas: F3, Fz, F4, FC1, FCz, FC2, C3, C1, Cz, C2, C4, CP1, CPz, CP2, P3, Pz, P4, O1, $\mathrm{Oz}, \mathrm{O} 2$. Horizontal and vertical EOG recordings were made using external bipolar channels positioned on the outer canthi of each eye as well as superior and inferior to the right eye. Reference electrodes were positioned on the mastoid bone behind the right and left ears. EEG signals were recorded at a sampling rate of $2048 \mathrm{~Hz}$. A trigger channel was used to mark the time of important events on the signal.

\section{EEG Preprocessing}

EEG data were preprocessed using MATLAB (The MathWorks) and EEGLAB toolbox (Delorme \& Makeig, 2004). Data were downsampled to $256 \mathrm{~Hz}$ and low-pass filtered at $30 \mathrm{~Hz}$ with no high-pass filtering. The average signal of the mastoid electrodes was used as a reference for all other electrodes. In Experiment 1, EEG epochs for the hand used for skipping (left vs. right) and action conditions (self-initiated vs. externally triggered) were separated, resulting in four different types of epochs: self-initiated skip with left hand, self-initiated skip with right hand, externally triggered skip with left hand, and externally triggered skip with right hand. EEG epochs in Experiment 2 were separated based on the action condition (self-initiated vs. externally triggered) and the availability of skip actions (unlimited vs. limited). Each epoch was 4 sec long, ranging from 3 sec before to 1 sec after skip action. Overlapping epochs, in which participants skipped earlier than $3 \mathrm{sec}$ from trial initiation were removed. Next, nonocular artifacts were removed from 
data using two methods of improbable data rejection (single channel $S D=6$, global $S D=2$ ) and extreme values rejection (upper threshold limit $=250 \mu \mathrm{V}$, lower threshold limit $=-250 \mu \mathrm{V})$. Following this, ocular artifacts components were extracted by independent component analysis and were identified and removed by visual inspection. Trials with artifacts remaining after this procedure were excluded by visual inspection. In keeping with our previous study (Khalighinejad et al., 2018), RP recordings were baseline corrected using a baseline of -5 to +5 msec with respect to action onset, avoiding any assumptions concerning the moment of $\mathrm{RP}$ initiation.

\section{EEG Analysis}

EEG analysis was conducted using MATLAB and FieldTrip toolbox (Oostenveld, Fries, Maris, \& Schoffelen, 2011). Mean RP amplitude across trials and variability of RP amplitudes across trials (measured by $S D$ ) were measured as dependent variables for each trial type. We previously showed that, in a bimanual task, EEG convergence is strongest in frontomidline electrodes (Khalighinejad et al., 2018). However, this topography might reflect either a midline cognitive process of deliberation or the summation of effector-related motoric activity linked to controlling both hands simultaneously. Our current design, based on blocked, unimanual skip responses, was designed to distinguish between these possibilities. The FCz electrode was chosen as a marker of effectorindependent cognitive processing, whereas C3 and C4 electrodes, positioned above the left and right motor cortex, respectively, were chosen as marking effectordependent processes in the contralateral motor cortices. First, cluster-based permutation tests were used to compare across-trial $S D$ between self-initiated and externally triggered conditions for each chosen electrode. The cluster-based permutation tests were performed using the following parameters: time interval $=[-2$ to $0 \mathrm{sec}$ relative to skip action], number of draws from the permutation distribution $=1000$.

In a subsequent step, we investigated whether EEG convergence is an effector-dependent or -independent process. EEG convergence was defined as the area between the $S D$ curve in self-initiated and externally triggered condition in a 2 -sec window before skip action onset. Individual EEG epochs were time-locked and baseline-corrected at action onset, making the across-trial standard deviation at the time of action necessarily zero. Using the difference between the self-initiated and externally triggered conditions would control for the reduction in variability that comes simply as a by-product of action-locked epoching. EEG convergence from C3 with left hand skips was averaged with EEG convergence from $\mathrm{C} 4$ with right hand skips to give the ipsilateral EEG convergence. Analogously, EEG convergence from C3 with right hand skips was averaged with EEG convergence from $\mathrm{C} 4$ with left hand skips to give the contralateral EEG convergence. EEG convergence from $\mathrm{FCz}$ with left hand skips was averaged with EEG convergence from $\mathrm{FCz}$ with right hand skips to give frontal-midline EEG convergence. As we planned to compare these neurocognitive processes between electrodes selected on the basis of previous studies, we used one-way repeatedmeasures ANOVA. The level of significance was set at .05 for all the analyses.

In the second experiment, we did not have a priori hypotheses about where in the brain "rationing" would be found, so we used a more conservative, exploratory approach. Briefly, nonparametric permutation tests across all electrodes from central areas (see EEG Recording section) were used to compare convergence between conditions in the second experiment. This approach avoids some of the arbitrary assumptions associated with electrode and time bin selection. The permutation tests were performed using the following parameters: time interval =

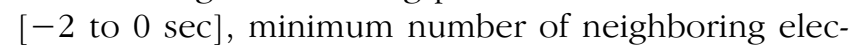
trodes required $=2$, number of draws from the permutation distribution $=1000$.

\section{RESULTS}

\section{Experiment 1: EEG Convergence Is an Effector-independent Process}

On average, participants ( $n=20)$ skipped waiting for coherent dot motion 63 times $(S D=3)$ with the left hand and 62 times $(S D=3)$ with the right hand in the selfinitiated condition. They skipped $62(S D=3)$ and 63 $(S D=3)$ times with the left and right hand, respectively, in response to the external cue. The mean waiting time before skipping was $6.68 \mathrm{sec}(S D=1.04)$ for the left hand and $6.91 \mathrm{sec}(S D=0.58)$ for the right hand in the selfinitiated condition (Figure $2 \mathrm{~A}$ and $\mathrm{B}$ ). This waiting time in the externally triggered condition was $7.22 \mathrm{sec}(S D=$ 1.11) and $7.46(S D=0.61)$ for the left and right hand, respectively. This confirms that our yoking procedure (see Methods) was successful (the slight difference between the waiting time in the self-initiated and externally triggered conditions is due to the time it takes for participants to react to external cue). For externally triggered trials, the RT to external cue (red fixation point) was $752 \mathrm{msec}(S D=76)$ and $742 \mathrm{msec}(S D=$ 89) for left and right hand, respectively. Participants gained, on average, an additional 125p $(S D=2.77)$ for skipping with the left hand and 125p $(S D=4.01)$ for skipping with the right hand. They further gained 125p $(S D=48.90)$ and $127 \mathrm{p}(S D=42.34)$ with the left and right hand, respectively, from correct responses to coherent dot motion. No significant difference was observed in any of the above behavioral measures between the left and right hand $(p>.27$ for all comparisons). 
Figure 2. Histogram of waiting time before skipping in self-initiated condition. In Experiment 1, participants could skip waiting by pressing the space bar with their left (A) or right (B) hand. In Experiment 2, participants used their both hands simultaneously to skip waiting, but in the "limited" condition (D) they were only allowed to make half the number of skips they made in the "unlimited" condition (C)
A

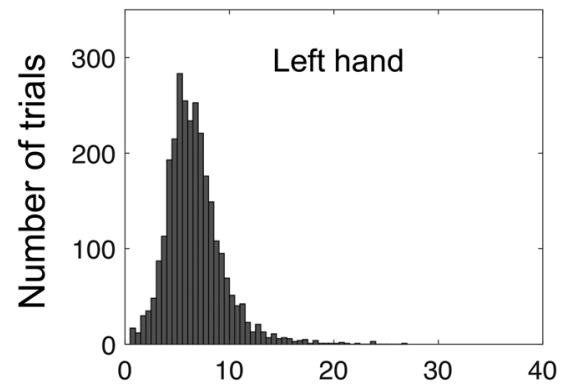

C

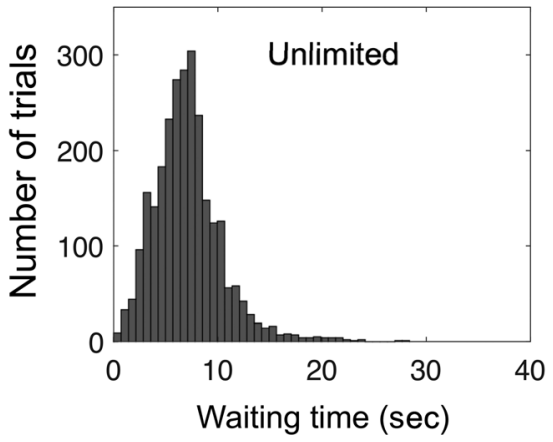

B

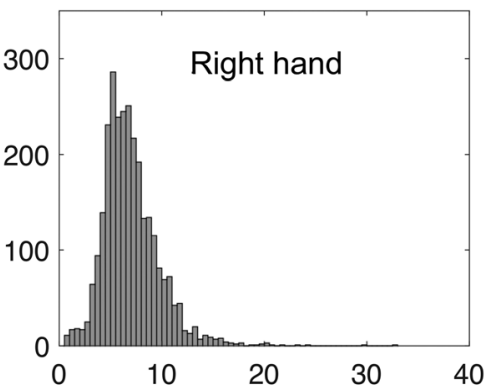

$\mathrm{D}$

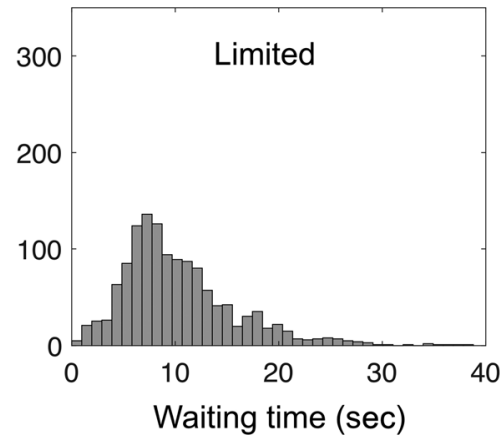

We next investigated whether RT to coherent dot motion was influenced by the hand assigned to skip response (e.g., how preparing to skip with the left hand influences the RT to rightward moving dots). We found that RT to dot motion direction was marginally lower when the hand used to respond to dot motion direction corresponded with the hand assigned for the skip response on that block, $F(1,19)=4.25, p=.05, \eta_{\mathrm{p}}^{2}=$ .18. This suggests that action initiation in response to an external cue (coherent dot motion) may be facilitated when precursor processes have already prepared for a self-initiated action (skip response).
Figure 3. EEG activity from the effector-independent electrode (FCz) before skip actions. The red and blue lines represent self-initiated and externally triggered skip conditions, respectively. Data are timelocked to the skip action (black vertical line), baseline-corrected in a 10-msec window around the skip. The average time of the skip instruction (fixation cross changing to red) in the externally triggered condition is shown as a gray vertical line. (A, B) Grand-averaged RP amplitude \pm SEM across participants for the skip responses with the left (A) and right (B) hand. (C, D) Standard deviation across trials averaged across participants \pm SEM for the skip responses with the left (C) and right (D) hand.

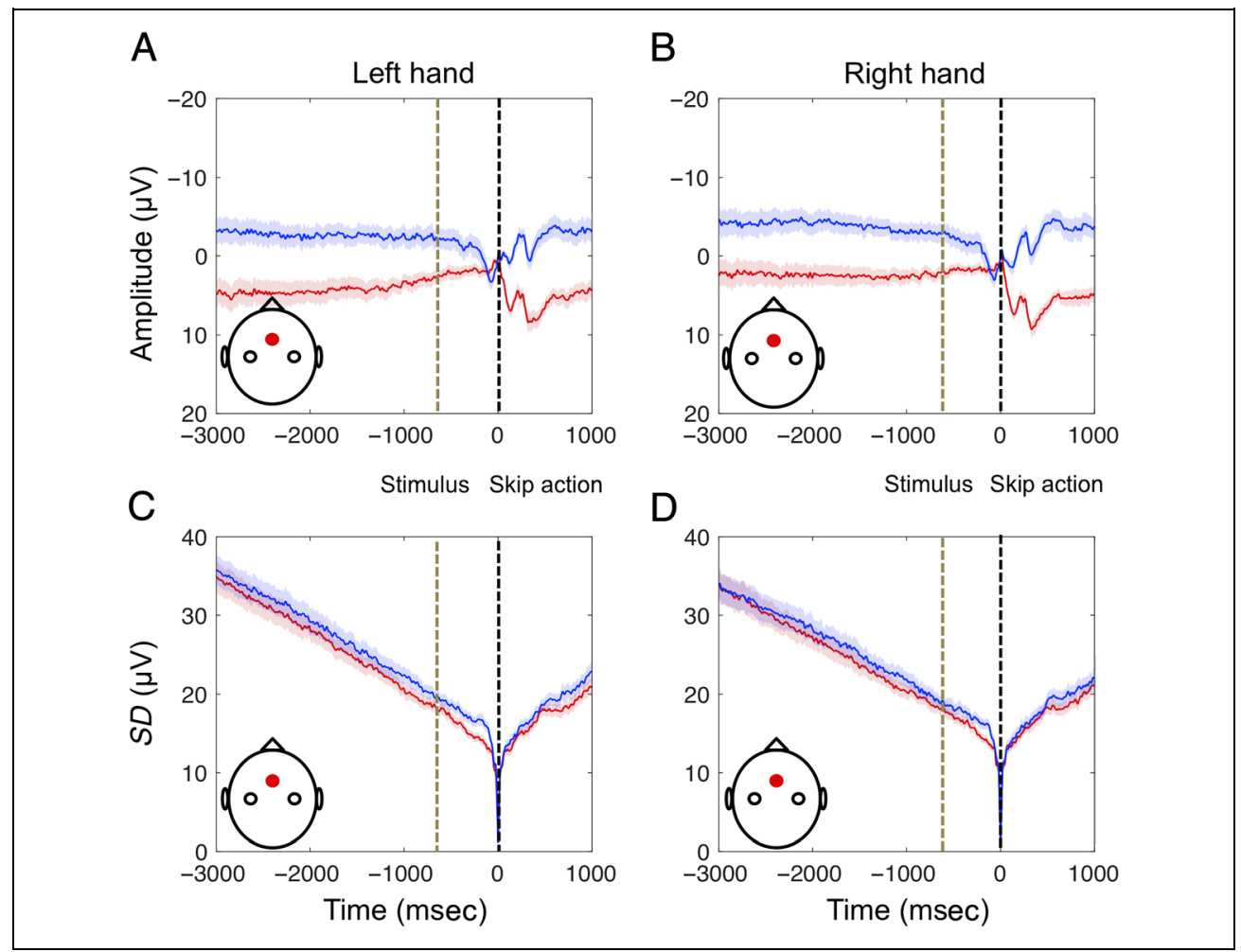


EEG data from 20 participants were preprocessed and pooled across the two sessions. Figures $3 \mathrm{~A}, \mathrm{~B}$ and $4 \mathrm{~A}, \mathrm{~B}$, $\mathrm{E}, \mathrm{F}$ show the grand-averaged RP for each trial type from the selected electrodes. The grand-averaged RP showed the expected negative-going shape for self-initiated action for both left and right hand skip responses and at all electrodes of a priori interest. As expected, RP was more prominent in electrodes contralateral to the skipping hand ( $\mathrm{C} 3$ for right hand skips [Figure $4 \mathrm{~B}$ ] and C4 for left hand skips [Figure 4E]).

We first aimed to replicate our pervious results (Khalighinejad et al., 2018) and to validate the use of EEG convergence as a reliable marker of self-initiated action. Thus, intertrial variability of RP amplitudes was
Figure 4. EEG activity from the effector-specific electrodes before skip actions. The red and blue lines represent self-initiated and externally triggered skip conditions, respectively. Format as in Figure 3. (A, B) Grand-averaged $\mathrm{RP}$ amplitude \pm SEM across participants from electrode $\mathrm{C} 3$ for the skip responses with the left (A) and right (B) hand. (C, D) Standard deviation across trials averaged across participants $\pm S E M$ for the skip responses from electrode $\mathrm{C} 3$ with the left (C) and right (D) hand. (E, F) Grand-averaged $\mathrm{RP}$ amplitude \pm SEM across participants from electrode $\mathrm{C} 4$ for the skip responses with the left (E) and right (F) hand. (G, H) Standard deviation across trials averaged across participants $\pm S E M$ for the skip responses from electrode $\mathrm{C} 4$ with the left $(\mathrm{G})$ and right $(\mathrm{H})$ hand.

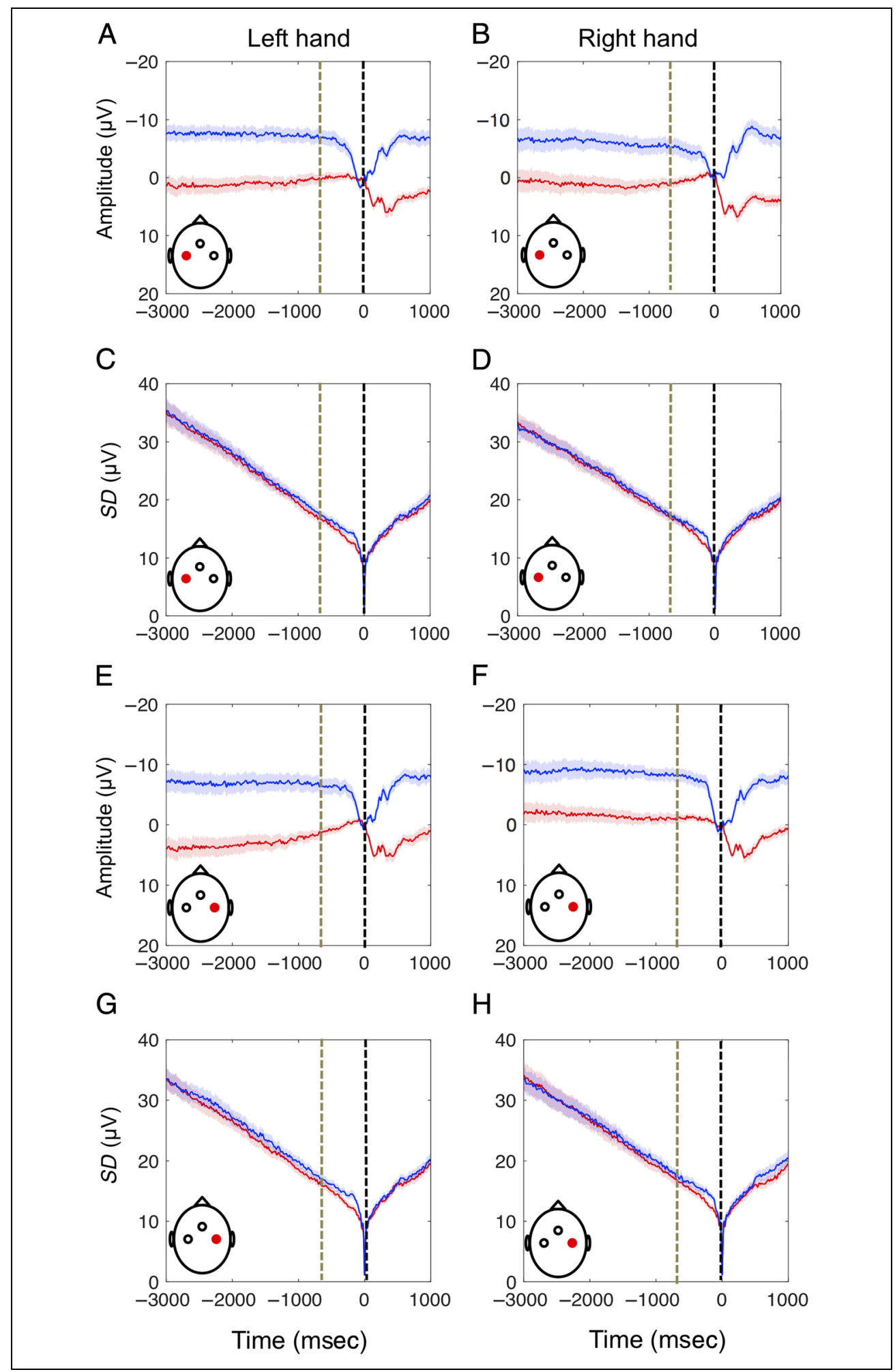


calculated for each trial type (Figures 3C, D and 4C, D, G, $\mathrm{H})$. The decrease in $S D$ is partly an artifact of time-locking and baseline correction. However, this artifact is common to the self-initiated and externally triggered conditions. Thus, the difference in convergence between the self-initiated and externally triggered conditions suggests a precursor process that precedes self-initiated action (see Khalighinejad et al., 2018, for details). Cluster-based permutation testing was used to investigate whether the $S D$ decrease before self-initiated skip action is significantly different from the $S D$ decrease before externally triggered skip action in our electrodes, in the latency range from -2 sec to the time of skip action onset (see Methods). Data from FCz (effector independent) showed significant EEG convergence for both left $(p=$ $.008, d=0.25)$ and right hand $(p=.006, d=0.26)$ skip responses. The same pattern was observed for effectordependent electrodes. Specifically, the convergence was significantly different for the left $(p=.018, d=$ $0.11)$ and right hand $(p=.01, d=0.11)$ data from C3 and the left $(p=.018, d=0.21)$ and right hand $(p=.006, d=0.15)$ data from $\mathrm{C} 4$. These findings confirm previous reports that neural activity before self-initiated actions gradually converges toward an increasingly stable pattern and thus could be used as a reliable marker of self-initiated action.

To ensure that the key cognitive factors in the task were balanced between self-initiated and externally triggered conditions, we also analyzed the mean and $S D$ of
EEG amplitude before stimulus-triggered responses to coherent dot motion (as opposed to skip responses) for each responding hand and each electrode. We did not observe any negative-going potential or differential EEG convergence before coherent dot motion $(p>$ .05 ; Figures 5 and 6 ). This suggests that the disproportionate drop in $S D$ before skip actions cannot be explained merely by a difference in background EEG, such as expectation of dot stimuli or temporal processing. That is, only those processes related to self-initiated action showed the distinctive EEG convergence, whereas general features of the context or block that were unrelated to the action event itself did not show any altered EEG variability.

After replicating our previous results, we aimed to investigate whether EEG convergence reflects an effectorindependent, cognitive process or an effector-specific motoric process. Based on the hand used for skipping, EEG convergence data (area between the $S D$ curve in self-initiated and externally triggered condition) was divided into three categories: ipsilateral, contralateral, and midline EEG convergence (see Methods for definitions). A one-way repeated-measures ANOVA showed a significant difference between these three categories, $F(2,38)=3.87$, $p=.030, \eta_{\mathrm{p}}^{2}=.17$ (Figure 7 ). The omnibus ANOVA was followed up by specific pairwise comparisons (no correction for multiple comparisons is required for this situation, following Fisher's least significant difference procedure; Meier, 2006). Specifically, midline EEG
Figure 5. EEG activity from the effector-independent electrode (FCz) before response to coherent dot motion. The red and blue lines represent self-initiated and externally triggered skip conditions, respectively. Format as in Figure 3. (A, B) Grand-averaged $\mathrm{RP}$ amplitude \pm SEM across participants for the responses with the left (A) and right (B) hand. (C, D) Standard deviation across trials averaged across participants \pm SEM for the responses with the left (C) and right (D) hand.

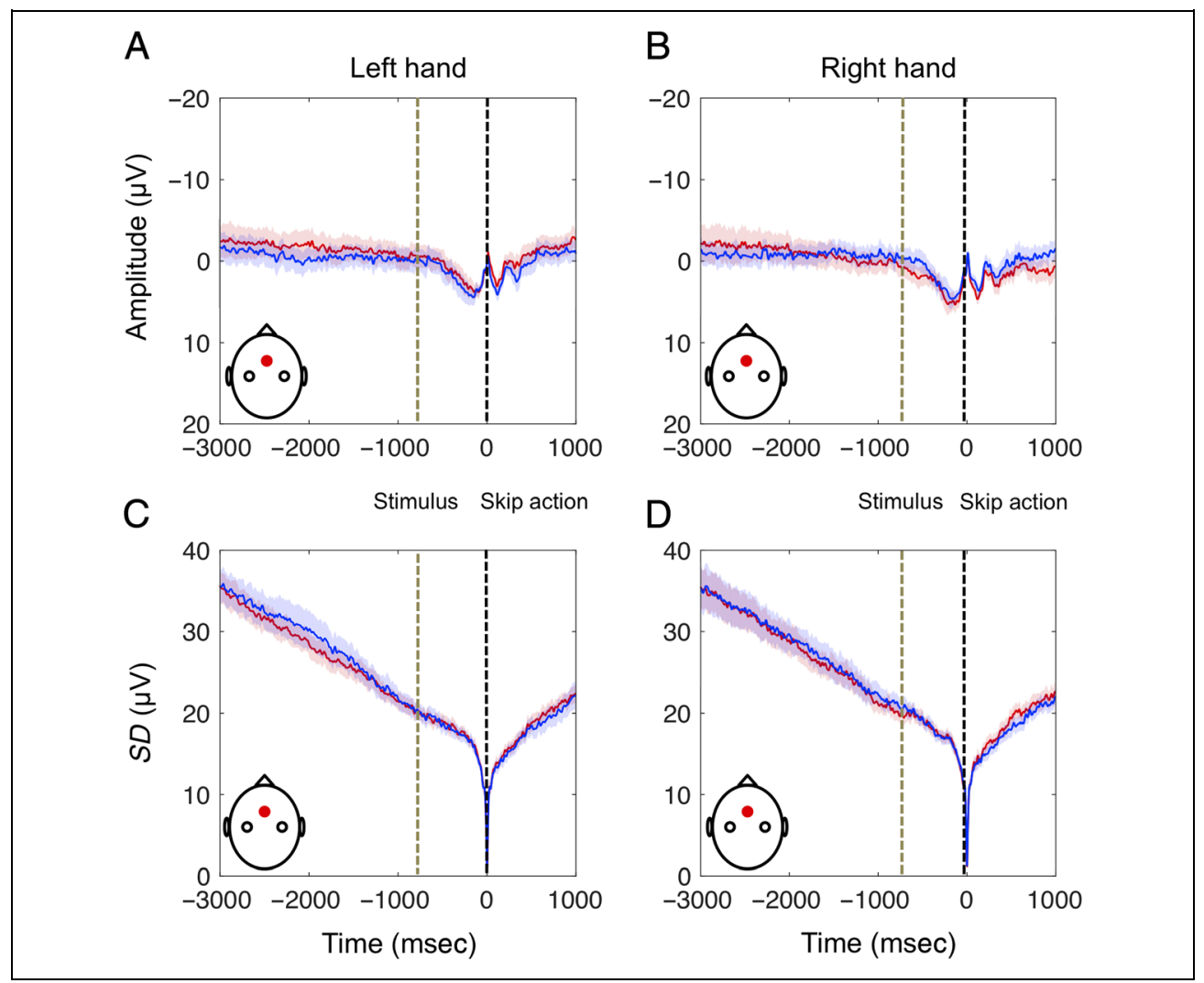


Figure 6. EEG activity from the effector-specific electrodes before response to coherent dot motion. The red and blue lines represent self-initiated and externally triggered skip conditions, respectively. Format as in Figure 4. (A, B) Grandaveraged RP amplitude \pm SEM across participants from electrode C3 for the responses with the left (A) and right (B) hand. (C, D) Standard deviation across trials averaged across participants \pm SEM for the responses from electrode $\mathrm{C} 3$ with the left (C) and right (D) hand. (E, F) Grand-averaged $\mathrm{RP}$ amplitude $\pm S E M$ across participants from electrode $\mathrm{C} 4$ for the responses with the left (E) and right (F) hand. (G, H) Standard deviation across trials averaged across participants $\pm S E M$ for the responses from electrode $\mathrm{C} 4$ with the left $(\mathrm{G})$ and right $(\mathrm{H})$ hand.
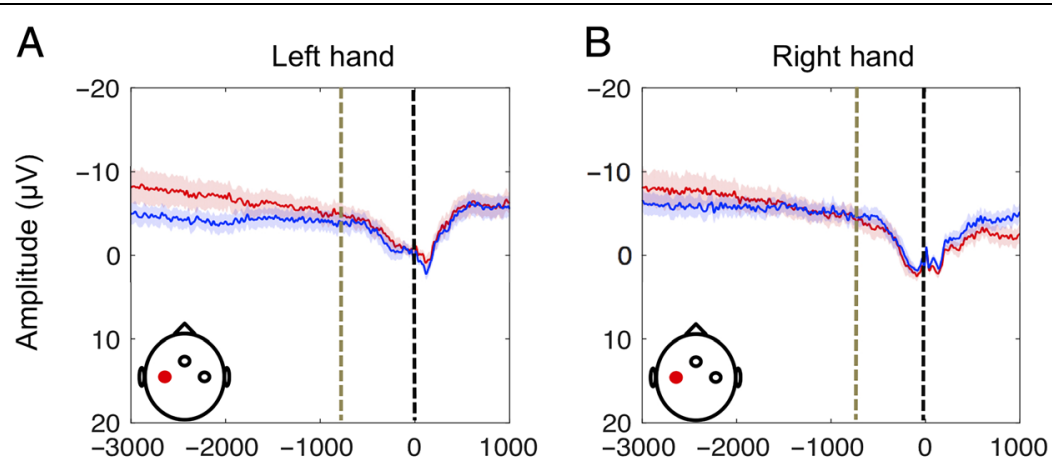

C

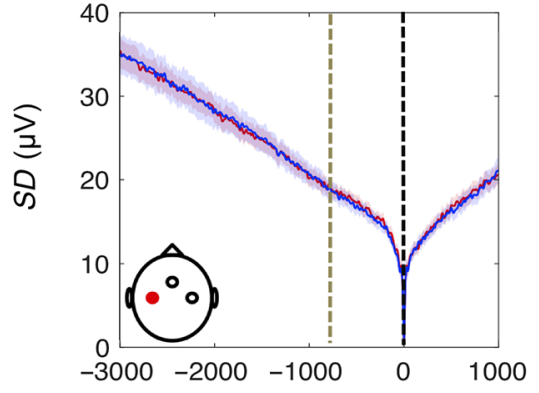

D

E

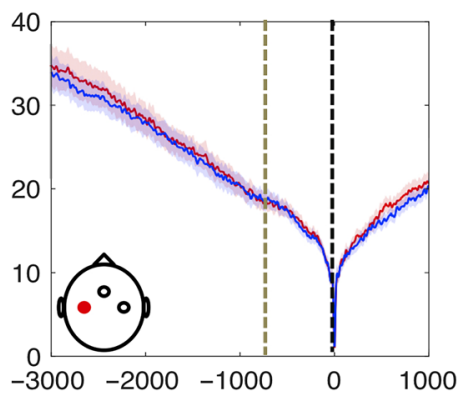

$\mathrm{F}$
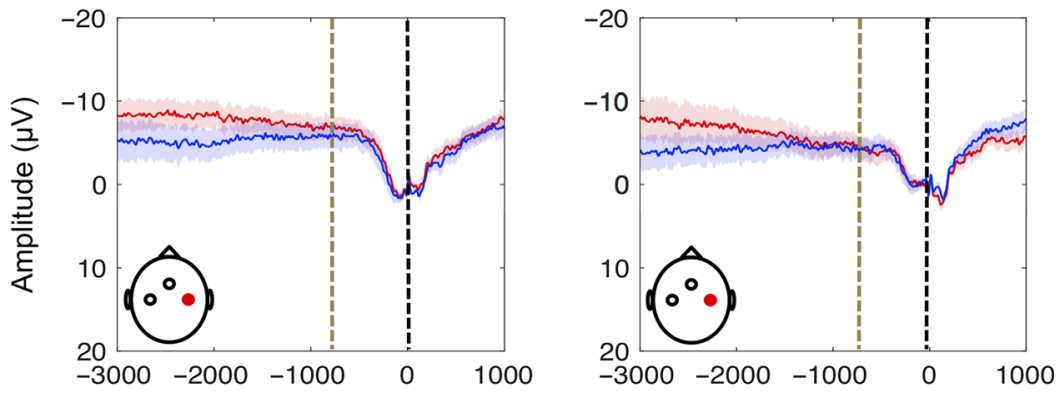

G

$\mathrm{H}$

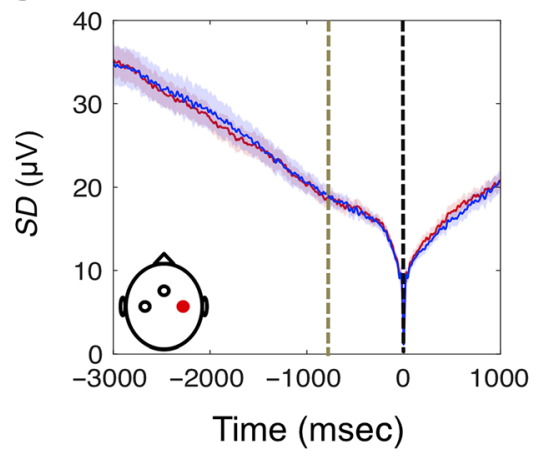

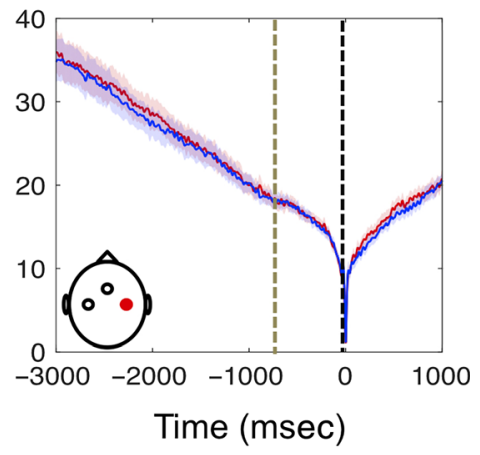

convergence was stronger compared with both ipsilateral, $t(19)=-2.60, p=.017, d=0.58$, and contralateral, $t(19)=-2.12, p=.048, d=0.47$, EEG convergence. In contrast, there was no difference between the contralateral and ipsilateral EEG convergence, $t(19)=0.24, p=.816$. These results suggest that the primary neurocognitive process measured by EEG convergence is not lateralized and does not depend on the specific effector used to perform a self-initiated action.

\section{Experiment 2: Deliberation About Self-Initiated Action Boosts Neural Precursor Processes}

If, as suggested by Experiment 1, our putative signal of self-initiated action is an effector-independent cognitive 


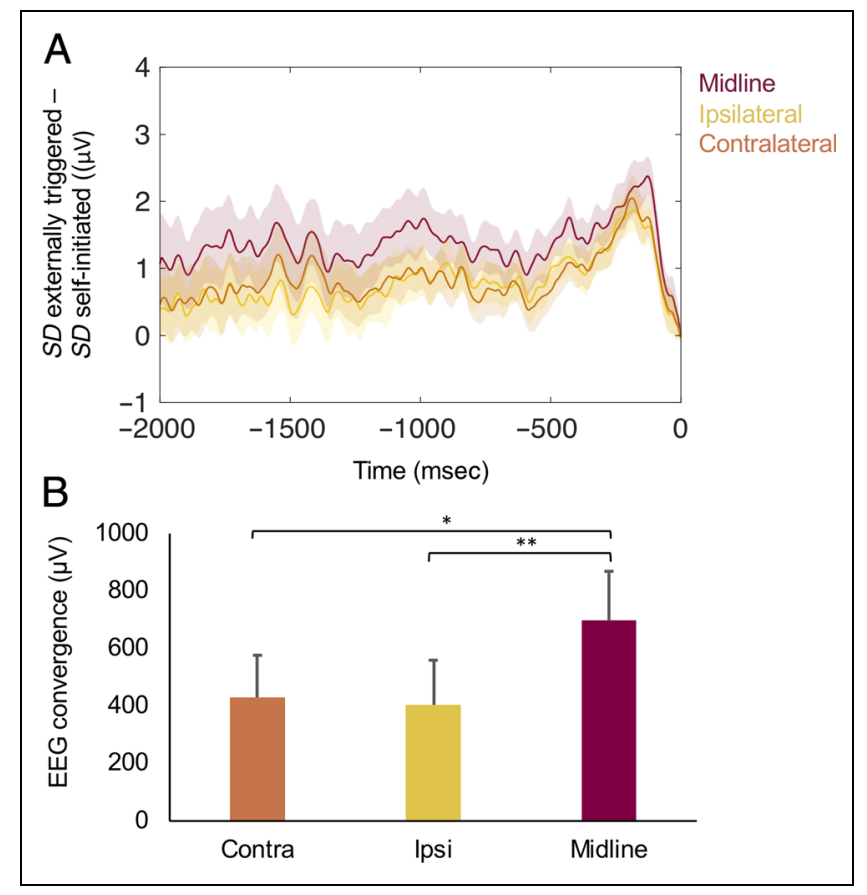

Figure 7. (A) The difference between standard deviation of externally triggered and self-initiated blocks for ipsilateral, contralateral, and midline EEG data (see Methods for definitions). (B) EEG convergence, defined as the area between the $S D$ curve in self-initiated and externally triggered condition, was significantly stronger for midline compared with ipsilateral and contralateral EEG data.

process, we would expect a stronger signal when this process is highlighted by key cognitive conditions that affect volition. In this experiment, we "rationed" volition by restricting the number of self-initiated actions that participants could use to skip long foreperiods. In the "unlimited" condition, participants could skip as many times as they wanted. On average, they skipped 123 times ( $S D=48)$. For the "limited" condition, they were instructed that they could make exactly half the number of skips they had previously made in the "unlimited" condition. On average, they made 59 skips $(S D=23)$. On average, participants waited $7.6 \mathrm{sec}(S D=1.7)$ before skipping in the self-initiated "unlimited" condition and $8.1 \mathrm{sec}(S D=1.8)$ in the externally triggered "unlimited" condition. For the "limited" condition, they waited $11.3 \mathrm{sec}$ $(S D=2.2)$ and $11.8(S D=2.2)$ in the self-initiated and externally triggered action blocks, respectively. Waiting time before skipping was significantly longer and had a wider distribution in self-initiated "limited" compared with "unlimited" condition, $t(18)=10.33, p<.001, d=$ 2.36 (Figure $2 \mathrm{C}, \mathrm{D}$ ). This suggests that rationing the number of "skip" actions encouraged careful planning of self-initiated actions and a deliberate decision to use skip actions only where they were most valuable-that is, when the wait for dot motion onset was particularly prolonged. Importantly, we did not find any evidence for differences in the RTs to coherent dot motion between the "limited" (848 msec, $S D=167$; averaged across both hands) and "unlimited" (887 msec, $S D=197$; averaged across both hands) conditions, $t(18)=1.8, p=.09$. Thus, limiting the number of skip responses does not seem to affect effector-specific processes.

EEG data from 19 participants were preprocessed. The number of skip responses in the "limited" condition was restricted by instruction to half the number of skip responses that the participant had previously made in the "unlimited" condition. To control for this difference when measuring intertrial variability, a subset of trials, equal to the number of skip responses of each participant in the "limited" condition, was randomly extracted from the unlimited condition. To avoid any bias in selection of the samples, 1000 samples were randomly extracted. Mean RP amplitude and variability of RP amplitudes across trials were measured within each sample. All subsequent EEG analysis of the "unlimited" condition was performed on the mean RP amplitude and mean variability across those 1000 samples. Figure 8A, B shows the grand-averaged RP for each trial type. The grandaveraged RP showed the expected negative-going shape for self-initiated action in both "limited" and "unlimited" conditions. The difference in EEG convergence between the self-initiated and externally triggered actions was significant in the "limited" condition $(p=.002, d=0.44$, cluster-based permutation test across central electrodes; Figure 8D) but not in the "unlimited" condition $(p=$ .11 , cluster-based permutation test across central electrodes; Figure 8C). Importantly, cluster statistics showed that this decrease in trial-to-trial variability was significantly more marked when the number of skip actions was limited compared with "unlimited" condition $(p=$ $.04, d=0.56$, cluster-based permutation test across central electrodes; Figure 8E). This suggests that the putative precursor signal of self-initiated action is intensified when participants are encouraged to plan and deliberate "when" to initiate a volitional action.

\section{DISCUSSION}

We measured the convergence of individual trial EEGs toward a stable RP-like pattern before self-initiated "skip" actions and before unpredictable external signals instructing a skip. We used the degree of convergence as a proxy for the neural processes underlying self-initiated voluntary action initiation. We found that this convergence was independent of the hand designated to execute the motor action and predominantly involved frontal midline structures (Experiment 1), suggesting it reflects cognitive preparation for volitional action, rather than motoric preparation for action execution. We also found that restricting the availability of self-initiated actions, which presumably led to enhanced deliberation before action initiation due to greater action value, boosted this convergence (Experiment 2). We conclude that EEG convergence before self-initiated action reflects a precursor neural process that involves deliberation and motivation 
Figure 8. EEG activity before skip actions. The red and blue lines represent self-initiated and externally triggered skip conditions, respectively. Data are time-locked to the skip action (black vertical line), baseline-corrected in a 10-msec window around the skip, and recorded from $\mathrm{FCz}$ electrode. The average time of the skip instruction (fixation cross changing to red) in the externally triggered condition is shown as a gray vertical line.

(A, B) Grand-averaged RP amplitude \pm SEM across participants for the unlimited (A) and limited (B) skip conditions. (C, D) Standard deviation across trials averaged across participants $\pm S E M$ for the skip responses in the unlimited (C) and limited (D) skip conditions. (E)

Topography of the difference in EEG convergence between limited and unlimited skip conditions. The time interval (sec) is indicated above each subplot.

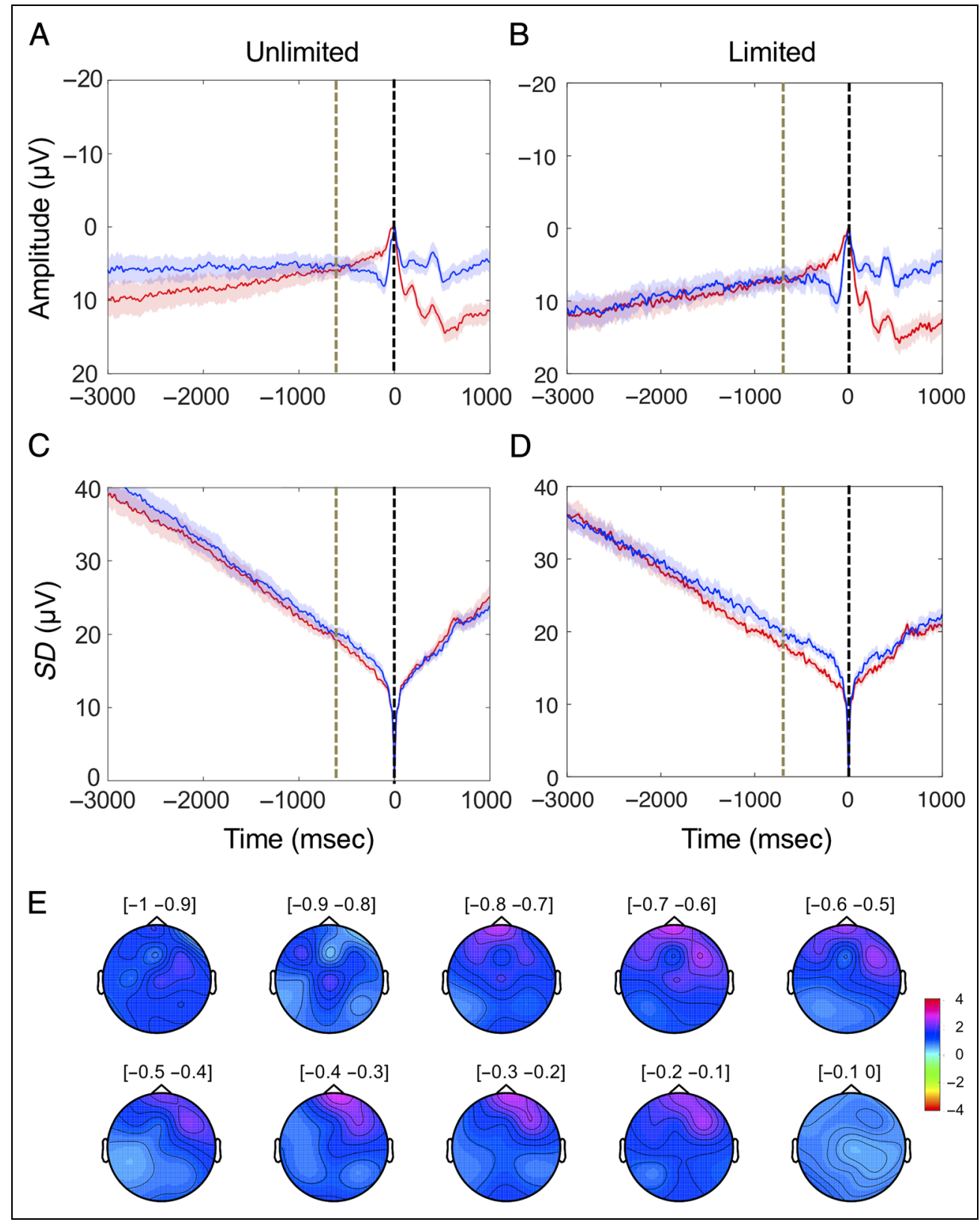

for action and not merely the motoric means of executing the action.

The common interpretation of the RP as a causal signal for voluntary action has been recently challenged. New models suggest that the decision of "when to move" in a self-initiated task is determined by stochastic fluctuation in neural activity (Schurger et al., 2012). Extending these new interpretations, we recently showed that selfinitiated actions are preceded by a gradual convergence in neural activity toward an increasingly stable pattern. Interestingly, this convergence could be modeled within a modified stochastic fluctuation framework, but only by assuming an additional process of neural noise regulation (Khalighinejad et al., 2018). In this study, by performing two separate experiments, we asked if convergence reflects a general, effector-independent cognitive process or an effector-dependent, lateralized motoric process. Experiment 1 aimed to investigate the origin in the brain of this convergence before self-initiated action. EEG variability decreased more markedly before self-initiated compared with externally triggered actions, replicating our previous findings (Khalighinejad et al., 2018). This convergence in EEG was observed at electrodes over the motor cortices and also at more frontal midline electrodes classically associated with RP. Importantly, control analyses ruled out the possibility that EEG convergence was merely some unknown contextual difference between the self-initiated and externally triggered action conditions. Coherent dot motion starts at a random, unpredictable time. Therefore, any general contextual difference, 
not related to action preparation, should be captured by EEG variability time-locked to responses to coherent dot motion-yet no such differences were found. This suggests that reduced variability in self-initiated skip conditions is linked to the impending action itself and not to any general difference in expectancy or task demands between the two conditions.

EEG convergence was stronger over effector-independent, midline electrodes (FCz electrode) compared with electrodes contralateral and ipsilateral to the hand used for skipping (C3 and C4 electrodes). This suggests that EEG convergence is frontally and bilaterally distributed. We cannot completely rule out the possibility that our midline electrode might also record effector-specific signals. For example, the SMA, which is thought to underlie the FCz electrode, is known to contain both effector-independent and effector-specific neurons (Nakayama, Yokoyama, \& Hoshi, 2015). We cannot know the exact contribution of each cell type to our surface EEG recordings. However, previous literature suggests that a substantial majority of SMA neurons are activated when participants move either the ipsilateral or contralateral hand (Kermadi, Liu, Tempini, Calciati, \& Rouiller, 1998; Tanji, Okano, \& Sato, 1987, 1988). Additionally, the timing and scalp location of the volitional skip EEG convergence shown in Figure 3 suggest that the convergence may arise upstream of the generators of LRP, which have been localized to the primary motor cortex and lateral premotor cortex (Haggard \& Eimer, 1999; Eimer, 1998). Thus, this precursor process of selfinitiated action appears to be independent of how the action is motorically expressed. Our results, therefore, distinguish an early cognitive decision to make a selfinitiated action from the later motoric computations regarding "how" to implement and execute that action. Our findings favor a serial and hierarchical evolution of self-initiated action, with a specifically cognitive stage of deliberation, that occurs in advance of execution-related stages. This contrasts with other models, in which interactive competitive inhibition between motor execution plans might, in itself, constitute the decision process (Cisek, 2012).

Recent models view action preparation and execution as two independent processes with distinct neural basis (Haith, Pakpoor, \& Krakauer, 2016). However, self-initiated action execution and preparation may not necessarily be serial processes that follow each other in a deterministic way but could rather be two independent overlapping processes, with the gradual flow of activity from the preparatory to execution state, as opposed to a sharp transition. For example, Orban de Xivry, Legrain, and Lefèvre (2017) measured RT during a virtual line bisection task in which participants used a robotic manipulandum to move a cursor smoothly through the middle of a target line that varied in its location and orientation. Movements to targets farther away from the starting position had shorter RTs than movements to closer targets. They also observed that a larger increase in movement curvature from the nearer to farther target was associated with a larger reduction in RT. They concluded that, if processed before movement onset, the cognitive demands of accurately planning the movement gave rise to an increase in RT. However, delaying accuracy planning during execution led to a substantial decrease in RT. They thus argued that preparation for voluntary action might overlap in time with movement execution, although the two processes may still be informationally independent. For instance, an overlap period between preparation and execution may explain why one can still veto an internally generated action even after the onset of action preparation (Schultze-Kraft et al., 2015).

Recently, Bozzacchi, Cimmino, and Di Russo (2016), studied the motor preparation of the two hands' movements in a task where participants had to reach congruent or incongruent targets. Motor-related cortical potentials showed that movements of both hands were programmed as a single motor plan, although they differentiated during movement execution depending on the target. A lack of lateralization in neural activity before movement onset, including in incongruent movement conditions in which each hand moved toward a different target, suggested that the two hands' actions were planned as a whole and not as separate movements. Interestingly, these authors used a self-paced task, where participants could freely choose when to initiate the movement. Thus, suggesting a possible link between the "single motor program" in their study and the "effector-independent precursor processes" that we identified.

Whereas Experiment 1 investigated the role of late, motoric processes, Experiment 2 focused instead on early, motivational processes for initiating voluntary action. We reasoned that, if our putative signal of selfinitiated action indeed represents an early process linked to the motivation and value of initiating action, we might be able to boost this signal by encouraging participants to plan and deliberate over when to act. Participants waited longer, and EEG convergence was stronger when they could only perform a limited number of skip actions compared with a condition with no such limit. This effect could be driven either by an exaggerated EEG convergence in the "limited" condition, as self-initiated actions become more deliberate and goaldirected, or by a diminished EEG convergence in the "unlimited" condition, as self-initiated actions become more frequent, more routine, and more habitual. Our design and analysis cannot directly tease apart the opposing effects of deliberative volition, on the one hand, and of habitual action, on the other. In our "unlimited" condition, reduction in intertrial variability of self-initiated actions was not significantly different from that for externally triggered actions, although the numerical direction was consistent with previous studies (Khalighinejad et al., 2018). One might have expected this reduction to be significant, as it is essentially a replication of our previous 
experiment. However, in this study participants knew that the "unlimited" condition would be followed by a "limited" condition, and this may have led them to increase the frequency of the skips in the "unlimited" condition. We may speculate that participants' self-initiated actions became more habitual as they became more frequent and then showed no more convergence or stability in the EEG pattern than control trials without self-initiated action. On this view, the distinctive pattern of neurocognitive activity before self-initiated action would reflect a specific process of deliberating whether or not to act now, and this process would be intensified when actions are limited.

Interestingly, a recent study compared neural precursors of action for arbitrary and deliberate decisions (MaOz, Yaffe, Koch, \& Mudrik, 2018). Although RPs were found for arbitrary decisions, they were absent in a condition involving value-based decisions. At first this may seem contrary to our findings. However, in that study, the mean RP was used as the precursor signal of action. We likewise found no difference between RPs in deliberate decisions of "limited" condition and habitual decisions of "unlimited" condition. But when using EEG convergence, rather than RP, as our putative signal of self-initiated action, we found evidence for stronger processing before action decisions in the "limited" condition. This finding suggests that EEG convergence reflects a general cognitive process that could be intensified by careful planning and deliberation.

In summary, we showed that EEG convergence is a robust and reliable marker of self-initiated voluntary action. Importantly, this convergence in neural activity is a neurocognitive process that is bilaterally distributed and is effector independent. The neural processes of selfinitiated action appear to be independent of how the action is motorically expressed but are sensitive to the reasons and motivation for initiating action. Cognitive models of intentionality propose a serial and hierarchical progression from prior intention to intention in action (Searle, 2008) or from distal intention to proximal and motor intention (Pacherie, 2008). Our finding shows that a consistent neural activity is associated with the earlier stages of this chain and not only with final motor execution.

\section{Acknowledgments}

This work was supported by the European Research Council Advanced Grant HUMVOL (grant 323943) and by the Leverhulme Trust (Ref. RPG-2016-378).

Reprint requests should be sent to Patrick Haggard, Institute of Cognitive Neuroscience, University College London, Alexandra House, 17 Queen Square, London WC1N 3AR, United Kingdom, or via e-mail: p.haggard@ucl.ac.uk.

\section{REFERENCES}

Anscombe, G. E. M. (2000). Intention (2nd ed.). Cambridge, MA: Harvard University Press.

Bozzacchi, C., Cimmino, R. L., \& Di Russo, F. (2016). The temporal coupling effect: Preparation and execution of bimanual reaching movements. Biological Psychology, 123, 302-309.

Brainard, D. H. (1997). The psychophysics toolbox. Spatial Vision, 10, 433-436.

Cisek, P. (2012). Making decisions through a distributed consensus. Current Opinion in Neurobiology, 22, 927-936.

Cunnington, R., Windischberger, C., Deecke, L., \& Moser, E. (2003). The preparation and readiness for voluntary movement: A high-field event-related fMRI study of the Bereitschafts-BOLD response. Neuroimage, 20, 404-412.

Deiber, M. P., Honda, M., Ibañez, V., Sadato, N., \& Hallett, M. (1999). Mesial motor areas in self-initiated versus externally triggered movements examined with fMRI: Effect of movement type and rate. Journal of Neurophysiology, 81, 3065-3077.

Delorme, A., \& Makeig, S. (2004). EEGLAB: An open source toolbox for analysis of single-trial EEG dynamics including independent component analysis. Journal of Neuroscience Methods, 134, 9-21.

Desantis, A., Waszak, F., \& Gorea, A. (2016). Agency alters perceptual decisions about action-outcomes. Experimental Brain Research, 234, 2819-2827.

Desantis, A., Waszak, F., Moutsopoulou, K., \& Haggard, P. (2016). How action structures time: About the perceived temporal order of action and predicted outcomes. Cognition, 146, 100-109.

Eimer, M. (1998). The lateralized readiness potential as an on-line measure of central response activation processes. Behavior Research Methods, Instruments, \& Computers, 30, 146-156.

Faul, F., Erdfelder, E., Lang, A. G., \& Buchner, A. (2007). G*Power 3: A flexible statistical power analysis program for the social, behavioral, and biomedical sciences. Behavior Research Methods, 39, 175-191.

Gold, J., \& Shadlen, M. (2007). The neural basis of decision making. Annual Review of Neuroscience, 30, 535-574.

Haggard, P. (2008). Human volition: Towards a neuroscience of will. Nature Reviews Neuroscience, 9, 934-946.

Haggard, P., \& Eimer, M. (1999). On the relation between brain potentials and the awareness of voluntary movements. Experimental Brain Research, 126, 128-133.

Haith, A. M., Pakpoor, J., \& Krakauer, J. W. (2016). Independence of movement preparation and movement initiation. Journal of Neuroscience, 36, 3007-3015.

Jenkins, I. H., Jahanshahi, M., Jueptner, M., Passingham, R. E., \& Brooks, D. J. (2000). Self-initiated versus externally triggered movements. II. The effect of movement predictability on regional cerebral blood flow. Brain: A Journal of Neurology, 123, 1216-1228.

Kermadi, I., Liu, Y., Tempini, A., Calciati, E., \& Rouiller, E. M. (1998). Neuronal activity in the primate supplementary motor area and the primary motor cortex in relation to spatio-temporal bimanual coordination. Somatosensory $\&$ Motor Research, 15, 287-308.

Khalighinejad, N., Schurger, A., Desantis, A., Zmigrod, L., \& Haggard, P. (2018). Precursor processes of human self-initiated action. Neuroimage, 165, 35-47.

Kornhuber, H. H., \& Deecke, L. (1965). Hirnpotentialänderungen bei Willkürbewegungen und passiven Bewegungen des Menschen: Bereitschaftspotential und reafferente Potentiale. Pflüger's Archiv für die gesamte Physiologie des Menschen und der Tiere, 284, 1-17.

Kornhuber, H. H., \& Deecke, L. (1990). Readiness for movement-The Bereitschafts potential-story. Current Contents Life Sciences, 33, 14.

Kutas, M., \& Donchin, E. (1980). Preparation to respond as manifested by movement-related brain potentials. Brain Research, 202, 95-115. 
Libet, B., Gleason, C. A., Wright, E. W., \& Pearl, D. K. (1983). Time of conscious intention to act in relation to onset of cerebral activity (readiness-potential). The unconscious initiation of a freely voluntary act. Brain: A Journal of Neurology, 106, 623-642.

Maoz, U., Yaffe, G., Koch, C., \& Mudrik, L. (2018). Neural precursors of decisions that matter-An ERP study of deliberate and arbitrary choice. BioRxiv, 097626. https://doi.org/10.1101/097626.

Meier, U. (2006). A note on the power of Fisher's least significant difference procedure. Pharmaceutical Statistics, 5, 253-263.

Murakami, M., Vicente, M. I., Costa, G. M., \& Mainen, Z. F. (2014). Neural antecedents of self-initiated actions in secondary motor cortex. Nature Neuroscience, 17, 1574-1582.

Nakayama, Y., Yokoyama, O., \& Hoshi, E. (2015). Distinct neuronal organizations of the caudal cingulate motor area and supplementary motor area in monkeys for ipsilateral and contralateral hand movements. Journal of Neurophysiology, 113, 2845-2858.

Oostenveld, R., Fries, P., Maris, E., \& Schoffelen, J. M. (2011). FieldTrip: Open source software for advanced analysis of MEG, EEG, and invasive electrophysiological data. Computational Intelligence and Neuroscience, 2011. https://doi.org/10.1155/2011/156869.

Orban de Xivry, J. J., Legrain, V., \& Lefèvre, P. (2017). Overlap of movement planning and movement execution reduces reaction time. Journal of Neurophysiology, 117, 117-122.

Pacherie, E. (2008). The phenomenology of action: A conceptual framework. Cognition, 107, 179-217.
Passingham, R. E. (1987). Two cortical systems for directing movement. Ciba Foundation Symposium, 132, 151-164.

Passingham, R. E., Bengtsson, S. L., \& Lau, H. C. (2010). Medial frontal cortex: From self-generated action to reflection on one's own performance. Trends in Cognitive Sciences, 14, 16-21.

Pereboom, D. (2011). Free will skepticism and meaning in life. In R. Kane (Ed.), The Oxford handbook of free will (2nd ed.). Oxford: Oxford University Press.

Schultze-Kraft, M., Birman, D., Rusconi, M., Allefeld, C., Görgen, K., Dähne, S., et al. (2015). The point of no return in vetoing self-initiated movements. Proceedings of the National Academy of Sciences, U.S.A., 113, 1080-1085.

Schurger, A., Mylopoulos, M., \& Rosenthal, D. (2016). Neural antecedents of spontaneous voluntary movement: A new perspective. Trends in Cognitive Sciences, 20, 77-79.

Schurger, A., Sitt, J. D., \& Dehaene, S. (2012). An accumulator model for spontaneous neural activity prior to self-initiated movement. Proceedings of the National Academy of Sciences, U.S.A., 109, E2904-E2913.

Searle, J. R. (2008). Intentionality: An essay in the philosophy of mind (1st ed.). Cambridge, UK: Cambridge University Press.

Tanji, J., Okano, K., \& Sato, K. C. (1987). Relation of neurons in the nonprimary motor cortex to bilateral hand movement. Nature, 327, 618-620.

Tanji, J., Okano, K., \& Sato, K. C. (1988). Neuronal activity in cortical motor areas related to ipsilateral, contralateral, and bilateral digit movements of the monkey. Journal of Neurophysiology, 60, 325-343. 